\title{
Shifts in outdoor activity patterns in the time of COVID-19 and its implications for exposure to vector-borne diseases in the United States.
}

Pilar Fernandez ( $\sim$ pilar.fernandez@wsu.edu )

Washington State University

Gebbiena Bron

University of Wisconsin-Madison

Pallavi Kache

Columbia University

Jean Tsao

Michigan State University

Lyric Bartholomay

University of Wisconsin-Madison

Mary Hayden

University of Colorado Colorado Springs

Kacey Ernst

University of Arizona

Kevin Berry

University of Alaska Anchorage

Maria Diuk-Wasser

Columbia University

\section{Research Article}

Keywords: COVID-19, outdoor activity, vector-borne diseases

Posted Date: May 26th, 2021

DOl: https://doi.org/10.21203/rs.3.rs-502309/v2

License: (c) (i) This work is licensed under a Creative Commons Attribution 4.0 International License.

Read Full License 


\section{Abstract}

Unintended consequences of human behavioral changes induced by governmental and individual responses to COVID-19 risk are yet to be fully realized. Herein, we evaluated changes in outdoor activity patterns during the spring and summer of 2020 vs. 2019 in the Northeast and Midwest United States. We used self-reported data from daily surveys available through a smartphone application, The Tick App. We simultaneously assessed changes in exposure to tick vectors in relation to changes in outdoor recreation. Outdoor activities and particularly peridomestic activities, increased by two-fold between years, while recreational activities in green spaces decreased by $70 \%$. Tick encounters increased by $30 \%$, and were mainly associated with outdoor activities and living in a rural county. Changes in outdoor recreation were associated with statewide stay-at-home orders in 2020 and persisted as out-of-home mobility increased. Our findings suggest that public health response should also address competing hazards from COVID-19 related outdoor exposure.

\section{Introduction}

The severity, complexity, and global reach of COVID-19 posed unprecedented challenges to public health practitioners. To combat the spread of SARS-CoV-2, starting in March 2020, millions of people in the United States were ordered to stay at home and voluntarily reduce in-person consumer traffic to avoid virus exposure. These actions were effective ${ }^{1-4}$, but also carried economic and social costs ${ }^{3,5}$.

Furthermore, stay-at-home orders only accounted for $7 \%$ of the observed reduction of consumer traffic ${ }^{6}$, as reductions were mainly driven by voluntary avoidance behaviors associated with fear of infection. The full consequences of the governmental and individual responses to the COVID-19 pandemic on societal well-being have yet to be fully understood.

The COVID-19 pandemic and associated governmental and individual responses have had a profound impact in the detection, diagnosis and treatment of non-communicable ${ }^{7}$ and communicable diseases ${ }^{8,9}$ in 2020, including evidence of delayed diagnosis of tick-borne diseases in the United States ${ }^{10}$. Moreover, significant disruptions to essential health services persist as of April $2021^{11}$. At the same time, vectorborne diseases pose a growing threat to public health in the United States, with 650,000 cases reported between 2004 and $2016,75 \%$ of which were tick-borne diseases ${ }^{12}$. The epidemiology of vector-borne diseases is complex, with transmission to humans depending on human exposure and protective behaviors, as well as vector abundance, seasonality, infection status, distribution and behavior ${ }^{13-16}$. The likelihood of vector-human contact also varies depending on the eco-social setting, ranging from natural areas, urban green spaces and peridomestic environments. Thus, we predicted that changes in human behavior in response to COVID-19 risk and policies could modify the frequency, duration, and type of outdoor activities that people engage in (recreational or peridomestic), altering the likelihood of vectorhuman contact. 
In the United States, both green spaces and peridomestic environments are known risks areas for both tick- and mosquito-borne diseases ${ }^{17-19}$. However, time spent in natural environments provide great benefits for human physical and emotional well-being ${ }^{20-24}$. Thus, during stay-at-home orders implemented to reduce SARS-Cov-2 transmission, many parks and natural areas remained open to provide safe spaces for physical activity and reduce stress and mental fatigue. Yet, the overall impact of COVID-19 responses on outdoor activity patterns remains unclear. At a national scale there was an initial reduction in park use (between - 10 and - 20\%) at the beginning of the epidemic in the United States, but this varied by state ${ }^{25-27}$. Park use then increased over the spring and summer, but this apparent trend did not account for seasonal effects, since baseline values were estimated during the winter ${ }^{25}$. To our knowledge, no data are available regarding the impact of stay-at-home orders in 2020 on peridomestic outdoor activities, although an increasing trend in daily visits to nurseries, gardening and farm supply stores was registered from January 2020 through mid-April $^{6}$. However, seasonal effects might be contributing to these observed changes as well.

In this study, we asked whether governmental mandates designed to promote physical distancing and individual responses to the COVID-19 pandemic led to changes in people's outdoor activities during 2020. We asked if activity differed between natural and peridomestic settings, and if these changes altered exposure to ticks in endemic areas of the United States. Although mobility datasets derived from cell phone data are useful to understand coarse activity patterns ${ }^{28}$, individual-level data over time that incorporates adaptive behaviors (i.e., changes in the type of outdoor activities) can be more closely linked to potential changes in the exposure to vectors and corresponding risk of exposure to tick-borne (and other vector-borne) diseases ${ }^{29,30}$. Herein, we focused on a spatially-explicit individual approach using a novel smartphone application, The Tick App, to characterize outdoor activity patterns that could lead to tick exposure ${ }^{29}$. The app collects self-reported data on the frequency and type of outdoor activities and tick encounters through daily surveys, allowing us to assess behavior change patterns over time and the impacts of COVID-19 in 2020 on Tick App users.

\section{Methods}

The Tick App is a smartphone application that was developed as a research tool to understand human behaviors affecting tick exposure and engage the general public in tick prevention across the United States $^{29}$. The app uses epidemiological e-surveys and GPS capabilities on the smartphone to gather data about mobility and outdoor activity patterns. A one-time baseline survey collects data on the user's tick exposure risk factors, demographic information and primary residence. Participants are asked to complete daily 1-minute surveys for at least one week, in which they are asked about outdoor activities and tick encounters (Supplementary Text 1 and Supplementary Text 2). Enrollment strategies, user profiles of participants, and usage patterns have been previously described ${ }^{29,30}$. In April 2020, we updated the daily survey to include questions related to COVID-19 transmission mitigation measures (Supplementary Text 2). 


\section{Data analyses}

Analyses were conducted on user data from the Midwest and the Northeast, United States, which are Lyme disease endemic areas where most Tick App users are located ${ }^{29,30}$. Users from these regions accounted for $95 \%$ (65.8\% from the Midwest and 29.2\% from the Northeast) of all survey responses recorded during the spring and summer of 2019 and 2020. To compare outdoor activity patterns between 2019 and 2020, we estimated the daily proportion of users reporting any type of outdoor activity during the same time of year (April 1st to July 31st) and used generalized linear models (binomial GLM, link function: logit) at the group level (i.e., proportion of users who were active outdoors versus those who stayed indoors) to assess temporal variations (between years and months), adjusted for weather variables (daily mean temperature and occurrence of rainy days), county level urbanicity (i.e., rural, small/medium metro and large metro counties), and demographic characteristics of the users (gender and age). Detailed description of the models and variables used are described in Supplementary Methods, but briefly the response variable was coded for each day as a vector of $(\mathrm{N}$, Total- $\mathrm{N})$, where $\mathrm{N}$ corresponded to the number of people reporting outdoor activities and the Total corresponds to the total number of users completing a daily survey that day. Responses were analyzed at the group level (per day) because user engagement and reporting in the app was variable, limiting the analysis of individual responses over time. Given that the response variable was analyzed as a daily proportion of users (at the group level), independent variables were summarized from individual level responses into appropriate daily variables experienced by users reporting on a given day, namely: the daily proportion of reporting users that live in a rural county, that live in a small/medium metropolitan area, that live in a large metropolitan area, that are females, and the median age of users estimated for each day. Weather variables recorded for each user (county-level daily precipitation and mean daily temperature) were transformed into appropriate group-level variables per each day: the median of all mean daily temperatures experienced by reporting users and precipitation and the daily proportion of reporting users experiencing a rainy day (with more than $2.4 \mathrm{~mm}$ of rain coded as a 'rainy day' binary variable). Seasonality was adjusted by considering quadratic effects of temperature in the model (Supplementary Methods). Responses were also modeled separately for the daily proportion of users reporting outdoor activities in their yard (i.e., peridomestic activities) and in parks or natural areas (i.e., recreational activities in green spaces). To assess the robustness of our findings, we repeated analyses considering only returning users (those who completed surveys in both 2019 and 2020).

To explore the effects of COVID-19 mitigation measures on outdoor activity patterns, we assessed changes in the daily proportion of outdoor activities over time (for peridomestic, recreational and combined activities, both in green and grey spaces) from February 1 st to July 31 st, 2020, using a similar binomial GLM (link function: logit) than used in the interannual comparison. Instead of year and month, we included a dichotomous variable that accounted for the periods before and after stay-at-home orders (Supplementary Methods). We also considered the incidence of COVID-19 cases per 100.000 reported by county (i.e., locally) and nationwide per day and a daily, county-level Shelter-in-Place Index, which represents the change in the proportion of people staying home compared to baseline (February 6th to 
12th, 2020) ${ }^{31}$ (Supplementary Methods). Urbanicity, weather, and demographic variables were also included as explanatory variables for outdoor activity.

We explored self-reported impacts of COVID-19 on outdoor activities obtained from daily surveys in 2020. These responses were only available starting on April 28th, after an update in the Tick App. We created a binary variable ("yes/no") from responses about self-reported impact of COVID-19 on outdoor activities (Question 10 in Supplementary Text 2), and individual responses were analyzed using generalized linear mixed models (binomial GLMM, link function: logit) with user ID as a random variable. The independent variables considered included: Shelter-in-Place Index, the incidence of COVID-19 cases per 100,000 (locally and nationwide), urbanicity, gender, and age. We included month as an additional explanatory (ordinal) variable to analyze temporal changes between May and July 2020.

We used a binomial GLMM model to compare self-reported tick encounters ("yes/no") during the period when people are at greater risk for tick encounters (April-July) ${ }^{32}$ between 2019 and 2020, and assess its association with outdoor activities, adjusting for urbanicity, gender and age. We evaluated the model at the individual level rather than summarizing the daily responses across individuals to adjust for individual variation in tick exposure associated with reported preventative behaviors. We included an interaction between the month and year, as well as between month and outdoor activities to account for conditions that may have affected behavior at the population level. Data analyses were conducted in R statistical computing software ${ }^{33}$ and all models were checked for multicollinearity, misspecification, and performance (thoroughly described in Supplementary Methods). The code and data used in this manuscript are available at https://piliffq.github.io/outdooractivity_Covid/, except for individual level data to preserve users anonymity.

\section{Results}

Between April 1st and July 31st 2019, 994 users completed 7,597 daily surveys; during the same period in 2020, 1,329 users completed 9,380 surveys. Use of The Tick App was seasonal, mostly restricted to spring and summer (Fig. 1). Of the surveys completed in 2020, 21.1\% were completed by return users who had participated in 2019 (9.6\% of 2020 users, $n=128$ ). For the 2020 analysis of the effect COVID-19 mitigation measures, we extended the analysis period to February $1 \mathrm{st}$ to include data prior to the mandated stay-at-home order; 14 users and 355 additional surveys were included. When comparing the demographic characteristics of users from 2019 and 2020, we observed a 3-year difference in median age (Kruskal-Wallis, $P=0.05), 48(\mathrm{IQR}=26)$ in 2019 and 51 years old $(\mathrm{IQR}=26)$ in 2020; in both cases the age distribution was bimodal (Supplementary Fig. 1). In both years, more women completed daily surveys than men $\left(60.2 \%\right.$ vs. $\left.58.0 \%, \chi^{2}(1)=1.8, P=0.31\right)$. The distribution of users across a rural to urban gradient, however, was different between years $\left(\chi^{2}(2)=46.6, P<0.001\right)$ : in $2019,19.9 \%$ of users lived in rural counties, $48.8 \%$ lived in small and medium metropolitan areas and $31.3 \%$ lived in large metropolitan areas; in 2020 , the distribution of users was more even $(30.9 \%, 36.6 \%$ and $32.4 \%$ respectively) with a significant increase in users from rural counties. 


\section{Comparison of outdoor activity patterns between 2020 and 2019}

The daily proportion of users reporting any type of outdoor activity increased by two-fold in 2020 $\left(\mathrm{OR}_{\mathrm{adj}}=2.65, \mathrm{Cl}_{95}=2.33-3.00\right)$ compared to 2019 , after adjusting for urbanicity, demographic and weather variables (Supplementary Table 1), even though outdoor activities during the spring and summer were commonly reported by users on a daily basis both years (daily proportion of outdoor activities: median $=71.1 \%$, IQR $=12.3$ for 2019 and median $=86.7 \%$, IQR $=9.5$ for 2020) (Fig. 2). The two-fold increase was consistent during the period analyzed (the interaction term with month was non-significant). Weather variables influenced outdoor activities, where engagement in activities increased with mean daily temperature and decreased on rainy days. Adding a quadratic term for temperature improved model fit by capturing the temperature-driven seasonality of outdoor activities. The relationship between temperature and overall outdoor activities was concave up, meaning that outdoor activities increased with temperatures at the beginning of the spring but reached a plateau at high mean daily temperatures (e.g., in July) (Fig. 2 and Supplementary Table 1). Outdoor activities also decreased with the proportion of users living in large metropolitan areas.

Similar results were observed when analyzing the daily proportion of users reporting peridomestic activities, which doubled in $2020\left(\mathrm{OR}_{\mathrm{adj}}=2.26, \mathrm{Cl}_{95}=2.05-2.51\right)$ after adjusting for the same covariates (Fig. 2 and Supplementary Table 2). This increase in peridomestic activities in 2020 was most pronounced in April and May (Supplementary Table 3). By contrast, the daily proportion of recreational activities in parks and natural areas decreased in 2020 by $70 \%\left(\mathrm{OR}_{\mathrm{adj}}=0.26, \mathrm{Cl}_{95}=0.23-0.30\right)$ (Supplementary Table 4). Yearly and monthly interactions revealed opposing monthly trends for 2019 and 2020: in 2019, while recreational activities increased as the spring and summer progressed, the opposite was observed in 2020 (Supplementary Table 5). Although there was a decrease in recreational activities in green spaces in 2020, we also observed an increase in recreational activities in grey spaces (e.g., walking in sidewalks) although this variable was only recorded for May-July 2020 (Supplementary Fig. 2). Both peridomestic and recreational activities in green spaces increased with the proportion of users in rural areas as opposed to urban users. When only considering users who completed surveys both in 2019 and 2020, we observed similar shifts in the proportion of outdoor activities between years compared to the study population as a whole: an overall increase in outdoor activities, with an increase in peridomestic activities and a decrease in recreational activities in green spaces (Supplementary Tables 1 , 2 and 4).

\section{Impact of stay-at-home orders on outdoor activity patterns}

For 2020, we observed a two-fold increase in the daily proportion of users reporting any type of outdoor activity after statewide stay-at-home orders went into effect (April to July versus February and March, 2020) after adjusting for covariates $\left(\mathrm{OR}_{\mathrm{adj}}=2.01, \mathrm{Cl}_{95}=1.21-3.29\right)$ (Supplementary Table 6), and an even greater increase in peridomestic outdoor activities $\left(\mathrm{OR}_{\mathrm{adj}}=11.25, \mathrm{Cl}_{95}=6.73-19.55\right)$ (Supplementary 
Table 7). In contrast, the proportion of users reporting recreational activities in green spaces was $80 \%$ lower after the stay-home orders went into effect $\left(\mathrm{OR}_{\mathrm{adj}}=0.19, \mathrm{Cl}_{95}=0.12-0.30\right)$ (Supplementary Table 8). After adjusting for the effect of the statewide stay-at-home orders, the county-level Shelter-inPlace Index as a measure of out-of-home mobility restrictions (i.e., higher SIP means lower out-of-home mobility, Supplementary Fig. 4) was negatively associated with the proportion of users reporting outdoor activities overall and peridomestic activities in particular. In other words, as stay-at-home orders and human risk mitigation behaviors relaxed over time and out-of-home mobility increased (i.e., a decrease in the Shelter-in-Place Index, Supplementary Fig. 4), both the proportion of users reporting any type of outdoor activities and peridomestic activities increased (Supplementary Tables 6 and 7). However, this index was not associated with reported recreational activities in green spaces, which remained unchanged despite increased out-of-home mobility over time (Supplementary Table 8). We also observed that neither the national nor the local (county-level) incidence of COVID-19 cases were associated to the proportion of users reporting outdoor activities overall or recreational activities, but the proportion of users reporting peridomestic activities were inversely associated with increased COVID-19 incidence rates mainly at the local level (Supplementary Tables 6,7 and 8). This inverse association was particularly evident towards the June and July where cases drastically increased while the Shelter-in-Place Index remained constant (Fig. 2A vs. Supplementary Fig. 4). Recreational activities in green spaces were mostly affected by weather variables (mean daily temperature and rain), while peridomestic activities were only affected by rain but not significantly associated with mean daily temperature. The relationship between temperature and recreational activities in green spaces was convex down, meaning that the proportion of users reporting recreational activities was highest at cooler mean daily temperatures in 2020 (inversely associated); however, as daily mean temperatures increased, the association between temperature and the proportion of recreational activities in green spaces was attenuated (Supplementary Table 8). Regarding urbanicity levels, we observed that the proportion of peridomestic activities decreased significantly with both the proportion of reporting users living in rural and large metropolitan counties, possibly indicating that the increase in peridomestic activities was mostly driven by users living in small and medium metropolitan areas (Supplementary Table 7). No effect of urbanicity was observed for recreational activities in green spaces.

\section{Self-reported impact of COVID-19}

When asked about the impact of COVID-19 mitigation measures on outdoor activities on a daily basis (Question 10 in Supplementary Text 2), the majority of responses indicated no impact (Fig. 3A; Supplementary Table 9). Among individuals who reported an impact, the most frequent response was that they spent more time in their yard, however the distribution of responses changed throughout the study period $\left(X 2\right.$ test, $\left.X^{2}(16)=62.2, p<0.001\right)$ (Fig. 3A). The likelihood of reporting an impact on outdoor activities also varied by location and user demographics. The likelihood of reporting an impact increased with the Shelter-in-Place Index, if users lived in a metropolitan area as opposed to a rural county, and if they were female (Supplementary Table 10). For users who reported not recreating in parks or natural areas on a given day (Question 12 in Supplementary Text 2), most did not report reasons related to COVID-19 (Fig. 3B; Supplementary Table 9). Nonetheless, the reasons reported varied by month ( $\chi 2$ test, 
$\left.X^{2}(18)=113.7, p<0.001\right)$, and avoiding crowds was more commonly reported in April and May compared to other months, as were reports that users did not have park access, parks were closed, or stay-at-home orders were in place (Fig. 3B).

\section{Self-reported tick encounters}

Overall, self-reported tick encounters increased in 2020 (15.5\%) compared to 2019 (11.6\%) (OR $\mathrm{adj}=1.36$, $\mathrm{Cl}_{95}=1.12-1.64$ ) between April 1st and July 31st (Supplementary Table 11 and Fig. 4). Self-reported tick encounters increased if the user reported doing an outdoor activity the same day and decreased if the user lived in a metropolitan area compared to a rural county (Supplementary Table 11). When peridomestic or recreational activities in green spaces were analysed separately, we did not find a significant association with tick encounter for either variable. Although the overall probability of reporting a tick encounter was higher in 2020 compared to 2019, we observed monthly and yearly variation in the association between reporting an outdoor activity and a tick encounter (Supplementary Fig. 7). The increase in self-reported tick encounters was verified with picture submissions. The proportion of users submitting at least one picture increased from $33.9 \%$ in 2019 to $57.3 \%$ in 2020 . Although in both years the median number of ticks submitted per capita was one (median $=1$, IQR $=1$, range $=1-74$ in 2020 and median $=1, I Q R=0$, range $=1-24$ in 2019), the number of ticks per capita submitted was higher in 2020 vs 2019 (Mann-Whitney test, $P<0.001$ ) as well as the proportion of users submitting more than one tick picture for identification ( $50.5 \%$ in 2020 vs. $37.1 \%$ in 2019). In 2020, between April and July, 1,560 pictures were submitted from the Midwest and Northeast regions, $94.1 \%$ were ticks $(n=1,468)$, of which $69.1 \%$ were found on a person. During the same period in 2019,434 were submitted from those regions, $92.0 \%$ were ticks $(n=400)$ of which $67.5 \%$ were found on a person.

\section{Discussion}

In this study, we observed an increase in self-reported tick encounters during the spring and summer of 2020 compared to the same time period in 2019 , and an increase in outdoor activities overall. The timing of the initial shift in outdoor activities coincides with COVID-19 stay-at-home orders in the United States, suggesting changes in tick exposure were influenced by 2020 COVID-19-driven behavioral changes. The comparison between pre- and amid-pandemic conditions (before and after March 2020) was enabled by the availability of a baseline dataset encompassing $\sim 7000$ responses from $\sim 1000$ users each year from the Tick App, a smartphone application designed to capture human behavioral risk factors associated with tick encounters. Although previous studies have shown an overall increase in outdoor activities in other countries in the Global North ${ }^{34,35}$ and even locally in the United States ${ }^{36}$, this is the first study to use individual-level data to show a two-fold increase in outdoor activities and a $30 \%$ increase in self-reported tick encounters in 2020 compared to 2019. Moreover, we showed a shift towards peridomestic activities compared to recreational activities in green spaces, the latter decreasing by $70 \%$ in the spring and summer of 2020 . 
Throughout the COVID-19 pandemic, outdoor activities and gatherings have been encouraged over indoor activities in order to curb SARS-CoV-2 transmission ${ }^{37}$. However, accessibility to public green spaces, differences in competing hazards (e.g. ticks, mosquitoes, rodents) between public green spaces and peridomestic settings, and risk perception (for COVID-19 transmission, and vector- or rodent-borne diseases), among other factors may have influenced not only the frequency of outdoor activities, but also where people decided to conduct their activities (peridomestic vs. green spaces). This study stands apart from previous assessments of outdoor activity during the COVID-19 pandemic by using individual-level information about shifts in outdoor activity patterns and self-protection efforts. Other studies have relied on aggregated mobility data to assess changes in park visitation as a proxy for outdoor recreation ${ }^{35,38-40}$. Individual-level data is essential to fully assess the risk of tick-and other vector-borne diseases, which depends on the background risk (e.g. vector density), exposure (e.g. the type, location and intensity of outdoor activities), and self-protection efforts (e.g. the use of repellents) ${ }^{16,17,41}$. Behavioral choices related to exposure and self-protection are made through decision-making processes where individuals make complex risk assessments in the face of tradeoffs between mitigating competing hazards. To identify how an individual adapts to changing risk, data collection must explicitly measure the behaviors at multiple time points and scenarios (i.e., changes in the type of outdoor activities). Another strength of the study is that we distinguished between activities undertaken in peridomestic environments vs. green spaces. Because unidentified mobility data cannot distinguish between yards and indoor locations, such datasets completely miss peridomestic activities.

The overall increase in any type of outdoor activity during the spring and summer of 2020 compared to 2019 (pre-pandemic) found in this study (after adjusting for weather and demographic covariates) may have resulted in increased exposure to vectors (ticks and mosquitoes) during the COVID-19 pandemic. This hypothesis is supported by the observed increase in self-reported tick encounters in 2020, verified by picture submissions through the smartphone app. At the individual level, we found that self-reported tick encounters were associated with engagement in outdoor activities, which provides further evidence for the link between increased outdoor activities and tick exposure. However, when evaluating the association of self-reported tick encounters and the type of activity (peridomestic activities or recreational activities in green spaces), no significant association was found with either type. The fact that the engagement in any type of outdoor activities was associated with a reported tick at the individual level but not for a specific type of activity could be explained by similar tick abundances in both settings, although no available data is available to assess this. Furthermore, ticks' prolonged attachment and increased likelihood of detection as ticks engorge creates uncertainty on the date when the tick was first attached and first spotted, making it difficult to match the tick to the specific activity that resulted in the exposure. Additionally, in the app, users can report several activities on the same day, further hindering the match with the tick report. Nonetheless, the group-level shift towards peridomestic activities and increased tick exposure indicates either an increase in the overall tick population or increased exposure in those settings where participants are less inclined to use preventative measures against tick bites ${ }^{30}$. A similar pattern was observed for mosquito-borne disease in two studies on dengue infection: i) a simulation model found that COVID-19 stay-at-home measures (in which 70\% of the population restricted 
their mobility to their house) led to an increase in overall infections and increased clustering of cases at the household level ${ }^{42}$; and b) an empirical study using dengue case report data in Singapur found a $37.2 \%$ increase in the number of cases from expected baseline levels attributed to COVID-19 stay-at-home measures $^{43}$.

Our study population was overrepresented by outdoor enthusiasts ${ }^{29}$, which is a group that tends to be at a higher risk of tick exposure than the population at-large. This makes The Tick App users a suitable population to explore how COVID-19 might have shifted overall exposure and the context of the exposure (in green spaces vs. peridomestic). Overall, statewide stay-at-home orders had a strong effect on reducing out-of-home mobility in the United States, reflected by a $15.2 \%$ increase in the time spent at home after the policies took effect ${ }^{38}$. In our study, this was also reflected in an increase in peridomestic activities in 2020 compared to 2019 and pre- and post-stay-at-home orders that were implemented in March 2020. This effect was more pronounced immediately after the orders went into effect, even after adjusting for weather variables to account for seasonality effects. This increase in peridomestic activities was reflected in the self-reported impacts of COVID-19 on the user's outdoor activities: among individuals who reported an impact, the most frequent response was that they spent more time in their yard, particularly in April and May.

Recreational activities in green spaces on the other hand decreased significantly between years and preand post-stay-at-home orders, even after adjusting for weather variables. Increased park visitations revealed by Google Mobility Reports ${ }^{44}$ were used by two studies 39,40 as a proxy for changes in outdoor activities in these environments. However, these trends were found to be primarily driven by seasonality at the county-level, providing little empirical support to an effect of COVID-19 risk mitigation responses on outdoor recreation activities ${ }^{45}$. By comparing the same period in 2019 and 2020 and adjusting for weather variables to account for seasonality, we found a reduction in recreational activities, even after adjusting for urbanicity levels. Consistent with our findings, another logitudinal study that used questionnaires to survey outdoor enthusiasts in the United States ${ }^{46,47}$ at three different time points after stay-at-home measures took effect (between April and May), found a reduction in the frequency of outdoor activities and the distance traveled to partake in these activities. Additionally, a nationwide online survey in the United States examining the effects of the COVID-19 pandemic on outdoor recreation trips, showed an estimated $26 \%$ reduction in trips per participant to public outdoor recreation sites post-COVID19 , as compared to pre-COVID-19 trips ${ }^{48}$. The shift to backyard activities detected in our study was also found in a survey administered to avid birdwatchers worldwide (mostly located in the Global North), $85 \%$ of respondents reported that COVID-19 changed their birding behavior, increasing their time focused on this activity but shifting mostly to yard birding ${ }^{49}$. It should be noted that crowded conditions forced park closures off and on to mitigate COVID-19 transmission risk or offered limited capacity -i.e., the crowds were too large for proper distancing, which could have affected people's engagement in these activities and the distance traveled, particularly in higher urbanicity locations. However, in our study few study participants reported not engaging in recreational activities due to 'no access' to parks (Fig. 3B). As our 
study focused on two specific regions, these results cannot be generalized to the entire United States where activity patterns in recreational areas may have differed during the pandemic.

When taken together, our findings and those in the aforementioned studies, suggest a reduction in recreational activities in green spaces during the first couple of months of the COVID-19 pandemic after stay-at-home orders went into effect. Although our study suggests that the overall increase in any type of outdoor activities during the COVID-19 pandemic were driven mostly by an increase in peridomestic activities, we cannot disregard an increase in recreational activities in grey spaces (e.g., walking around the neighborhood, Supplementary Fig. 2); however, there is insufficient data to analyze the effects of COVID-19 on these types of recreational activities. Studies conducted in Canada and the United States showed that, in the first month of the pandemic, the majority of participants engaged in physical activity either in the home environment or in their neighborhood ${ }^{34,46}$.

When analyzing changes in activity patterns over time, we observed that changes prompted after the stay-at-home orders in effect persisted until the end of the period analyzed (July 31st), even after adjusting for seasonality effects. However, we also found that the Shelter-in-Place Index (used as a proxy for reduced out-of-home mobility), which decreased over time after the first month following the stay-athome others went into effect, was inversely associated with the daily proportion of peridomestic activities and was not associated with the daily proportion of recreational activities in green spaces. In other words, as stay-at-home orders and human risk mitigation behaviors relaxed over time and out-of-home mobility increased (i.e., a decrease in the Shelter-in-Place Index, Supplementary Fig. 4), both the proportion of users reporting any type of outdoor activities and peridomestic activities increased. The COVID-19 incidence rate both at the national and county level showed the same pattern: an inverse association with the proportion of users reporting peridomestic activities but no association with the proportion reporting recreational activities in green spaces. This inverse association between local COVID-19 incidence and peridomestic activities was particularly evident towards the end of our study period in June and July 2020, when cases drastically increased (i.e. the "second wave") while the Shelter-in-Place Index remained constant (Fig. 2A vs. Supplementary Fig. 4). Our results suggest that there was a substantial shift in peridomestic activities prompted by the COVID-19 pandemic but, as the time progressed, activity patterns were less influenced by the COVID-19 risk mitigation responses directly (particularly for peridomestic activities) although did not return to pre-pandemic patterns. This temporal pattern was also observed in a national survey to outdoor enthusiasts; after the initial reduction in recreational activities in green spaces, there was a slight shift from neighborhood and city streets back to public lands in late May compared to early April, though still in lower proportions compared to pre-pandemic levels ${ }^{46}$. These results were supported by an increased percentage of respondents indicating that their new outdoor activity patterns would persist in the long-term towards the end of the study 46 .

Self-reported impacts of COVID-19 on recreational activities were not homogenous along a rural-to-urban gradient. While peridomestic activities were disproportionately higher in small and medium metropolitan areas and rural areas as compared to large metropolitan areas, the proportion of users reporting recreational activities in green spaces increased with the proportion of users in rural counties. Similarly, 
Rice et $\mathrm{al}^{47}$, found that outdoor enthusiasts residing in urban areas reduced their frequency of outdoor recreation participation. Accessibility to green spaces might have been a limiting factor in metropolitan areas across the United States. In contrast, in Norway, recreational outdoor activities increased in Oslo during the early spring after COVID-19 mitigation responses were implemented. This increase was associated with the wide availability of green spaces and corridors through the city, as well as the Norwegian principle of common access (allemannsretten) that grants anyone the right, within certain restrictions, to move freely across private and public uncultivated land (Outdoor Recreation Act, 1957) ${ }^{35}$. Thus, Oslo provides a range of opportunities for recreation along an urban-wilderness continuum that may not be available in many large metropolitan areas in the United States. Nonetheless, variations within Oslo were also observed: "Activities were intensified in the urban periphery, while marginal increases and, in some areas, decreases were evident toward the city center for both pedestrian and cycling activities." ${ }^{35}$ In our study, self-reported tick encounters were mostly associated with users living in rural counties, where tick hazards tend to be higher than in more urbanized areas, indicating that humantick encounters depend on the tick distribution and density as well as human activity patterns that determine exposure, even without accounting for preventative measures such as tick repellents or protective clothing.

Our study has several limitations, as with any observational and longitudinal study. Our conclusions are limited to a population that tends to engage in outdoor activities frequently and cannot be generalized to the general population. Given that The Tick App is marketed as an app to better understand human exposure to ticks, we expect that people at greater risk of tick encounters and previous tick exposure use it more frequently, introducing self-selection bias. Moreover, our population is biased towards older ages, thus limiting conclusions for younger age groups; children and teenagers were not included. Follow-up time and surveys completed by users were highly variable, thus data had to be pooled to study temporal (daily) variations. In addition, the survey was slightly modified in 2020 to capture outdoor activities more accurately. However, new changes were rolled out by late April 2020 and we validated our results by comparing reporting activities between and after roll out of the new version of the app. Lastly, although we accounted for urbanicity levels, pooling users from different geographical areas in the Northeast and the Midwest might mask other aspects affecting outdoor activity patterns and tick encounters occurring at the local level. Nonetheless, this study capitalized on a unique dataset collected that captures human behavioral risk factors for a consistent geographic area over time, allowing us to study the effects of COVID-19 mitigation efforts on outdoor activities and tick-borne disease risk, compared to a prepandemic baseline. Unlike other studies conducted after the pandemic started, this dataset is not subject to recall bias. Moreover, the data encompassed reports by a subset of users who completed surveys in both years, providing robust validation of findings. Finally, a limitation of the study is that the size of the dataset precludes assessments of local changes in vector exposure or geographic-specific risk of vectorborne diseases ${ }^{29,30}$.

While the COVID-19 pandemic continues to represent a significant challenge to public health, we cannot neglect other health risks. In the United States, tick-borne disease cases have doubled during 2004-2016, 
and nine vector-borne human diseases have newly emerged during the last decade ${ }^{12}$. The full impact of the pandemic on the transmission of tick-borne, and other vector-borne diseases is yet to be fully realized. This study could provide valuable insights into potential underreporting of tick-borne disease cases during the COVID-19 pandemic ${ }^{10}$. Our results show increased self-reported tick exposure (particularly among outdoor enthusiasts) in 2020, but its association to disease risk is unknown. We speculate that this increase is influenced by the shifts in outdoor activity patterns found in our study population. However, we cannot disregard effects of an increase in the population of ticks or in awareness of tickborne disease risk resulting in a more diligent search for ticks after being outdoors. The links between outdoor recreation and wellbeing are well-established ${ }^{50}$ and particularly during the COVID-19 pandemic ${ }^{51}$, outdoor activities have been (and should be) encouraged as a way to mitigate transmission risk while providing safe spaces for social interactions. Nonetheless, other hazards occurring in outdoor settings should be taken into account. Our results highlight the need to balance public health resources to avoid neglecting the disease burden caused by tick-borne and other vector-borne diseases.

\section{Declarations}

\section{Acknowledgments}

We thank all participants for taking time to share their experiences and we thank everybody who promoted The Tick App. We thank the University of Wisconsin Center for Health Enhancement System Services for continued support of the Tick App and to the students, Midwest Center of Excellence for Vector-borne Disease summer fellows and colleagues, Megan Porter and Sandra Zapata for tick identification and Tick App communications.

\section{Funding}

This publication was supported by the Cooperative Agreements Number U01CK000509-01 and U01CK000505, funded by the Centers for Disease Control and Prevention, and the National Science Foundation's Dynamics of Integrated Socio-Environmental Systems (CNH2) program (Award \#1924061). Its contents are solely the responsibility of the authors and do not necessarily represent the official views of the Centers for Disease Control and Prevention, the Department of Health and Human Services or the National Science Foundation.

\section{References}

1. Flaxman, S. et al. Estimating the effects of non-pharmaceutical interventions on COVID-19 in Europe. Nature. 584, 257-261 (2020).

2. Kelso, J. K., Milne, G. J. \& Kelly, H. Simulation suggests that rapid activation of social distancing can arrest epidemic development due to a novel strain of influenza. BMC Public Health. 9, 117 (2009).

3. Haug, N. et al. Ranking the effectiveness of worldwide COVID-19 government interventions. Nature Human Behaviour. 4, 1303-1312 (2020). 
4. Jacobsen, G. D., Jacobsen, K. H. \& Statewide COVID-19 Stay-at-Home Orders and Population Mobility in the United States. World Med. Health Policy. 12, 347-356 (2020).

5. Maharaj, S. \& Kleczkowski, A. Controlling epidemic spread by social distancing: Do it well or not at all. BMC Public Health. 12, 679 (2012).

6. Goolsbee, A. \& Syverson, C. Fear, lockdown, and diversion: Comparing drivers of pandemic economic decline 2020. 23 https://www.nber.org/system/files/working_papers/w27432/w27432.pdf (2020).

7. World Health Organization. COVID-19 significantly impacts health services for noncommunicable diseases. https://www.who.int/news/item/01-06-2020-covid-19-significantly-impacts-health-servicesfor-noncommunicable-diseases.

8. Hogan, A. B. et al. Potential impact of the COVID-19 pandemic on HIV, tuberculosis, and malaria in low-income and middle-income countries: a modelling study. The Lancet Global Health. 8, e1132e1141 (2020).

9. Nikolayevskyy, V. et al. Impact of the COVID-19 pandemic on tuberculosis laboratory services in Europe.European Respiratory Journa/57, (2021).

10. Wormser, G. P., Jacobson, E. \& Shanker, E. M. Negative impact of the COVID-19 pandemic on the timely diagnosis of tick-borne infections. Diagn Microbiol Infect Dis. 99, 115226 (2021).

11. Second round of the national pulse survey on continuity of essential health services during the COVID-19 pandemic. https://www.who.int/publications-detail-redirect/WHO-2019-nCoV-EHScontinuity-survey-2021.1.

12. Rosenberg, R. et al. Vital Signs: Trends in Reported Vectorborne Disease Cases - United States and Territories, 2004-2016. Morb. Mortal. Wkly. Rep. 67, 496-501 (2018).

13. Finch, C. et al. Integrated Assessment of Behavioral and Environmental Risk Factors for Lyme Disease Infection on Block Island, Rhode Island. PloS one. 9, e84758 (2014).

14. Fischhoff, I. R., Keesing, F. \& Ostfeld, R. S. Risk Factors for Bites and Diseases Associated With BlackLegged Ticks: A Meta-Analysis. Am J Epidemiol. 188, 1742-1750 (2019).

15. Mead, P. et al. Risk factors for tick exposure in suburban settings in the northeastern United States. Ticks and Tick-borne Diseases. 9, 319-324 (2018).

16. Diuk-Wasser, M. A., VanAcker, M. C. \& Fernandez, M. P. Impact of Land Use Changes and Habitat Fragmentation on the Eco-epidemiology of Tick-Borne Diseases. J Med Entomol. https://doi.org/10.1093/jme/tjaa209 (2020).

17. Fischhoff, I. R., Bowden, S. E., Keesing, F. \& Ostfeld, R. S. Systematic review and meta-analysis of tickborne disease risk factors in residential yards, neighborhoods, and beyond. BMC Infectious Diseases. 19, 861 (2019).

18. Gujral, I. B., Zielinski-Gutierrez, E. C., LeBailly, A. \& Nasci, R. Behavioral Risks for West Nile Virus Disease, Northern Colorado, 2003. Emerg Infect Dis. 13, 419-425 (2007).

19. Eisen, R. J. \& Eisen, L. Spatial Modeling of Human Risk of Exposure to Vector-Borne Pathogens Based on Epidemiological Versus Arthropod Vector Data. Journal of Medical Entomology. 45, 181- 
192 (2008).

20. White, M. P. et al. Spending at least 120 minutes a week in nature is associated with good health and wellbeing. Sci. Rep. 9, 7730 (2019).

21. White, M. P. et al. Recreational physical activity in natural environments and implications for health: A population based cross-sectional study in England. Prev Med. 91, 383-388 (2016).

22. Kardan, O. et al. Neighborhood greenspace and health in a large urban center. Sci. Rep. 5, 11610 (2015).

23. Pearson, D. G. \& Craig, T. The great outdoors? Exploring the mental health benefits of natural environments. Front. Psychol.5, (2014).

24. Park, B. J., Tsunetsugu, Y., Kasetani, T., Kagawa, T. \& Miyazaki, Y. The physiological effects of Shinrin-yoku (taking in the forest atmosphere or forest bathing): evidence from field experiments in 24 forests across Japan. Environ Health Prev Med. 15, 18-26 (2010).

25. Google Mobility Trends: How has the pandemic changed the movement of people around the world? Our World in Data https://ourworldindata.org/covid-mobility-trends.

26. Google US Mobility report - March 2020. https://www.gstatic.com/covid19/mobility/2020-0329_US_Mobility_Report_en.pdf\#page=29\&zoom=100,48,88 (2020).

27. Google US Mobility report - April 2020. https://www.gstatic.com/covid19/mobility/2020-0426_US_Mobility_Report_en.pdf (2020).

28. Oliver, N. et al. Mobile phone data for informing public health actions across the COVID-19 pandemic life cycle. Sci. Adv. 6, eabc0764 (2020).

29. Fernandez, M. P. et al. Usability and Feasibility of a Smartphone App to Assess Human Behavioral Factors Associated with Tick Exposure (The Tick App): Quantitative and Qualitative Study. J. Med. Internet Res. 7, e14769 (2019).

30. Bron, G. M. et al. Context matters: Contrasting behavioral and residential risk factors for Lyme disease between high-incidence states in the Northeastern and Midwestern United States. Ticks and Tick-borne Diseases. 11, 101515 (2020).

31. SafeGraph. Shelter in Place Index: The Impact of Coronavirus on Human Movement. SafeGraph https://safegraph.com/data-examples/covid19-shelter-in-place/.

32. Gatewood, A. G. et al. Climate and Tick Seasonality Are Predictors of Borrelia burgdorferi Genotype Distribution. Appl Environ Microbiol. 75, 2476-2483 (2009).

33. R Core Team. R: A language and environment for statistical computing (R Foundation for Statistical Computing, 2020).

34. Lesser, I. A. \& Nienhuis, C. P. The Impact of COVID-19 on Physical Activity Behavior and Well-Being of Canadians. International Journal of Environmental Research and Public Health. 17, 3899 (2020).

35. Venter, Z. S., Barton, D. N., Gundersen, V., Figari, H. \& Nowell, M. Urban nature in a time of crisis: recreational use of green space increases during the COVID-19 outbreak in Oslo, Norway. Environ. Res. Lett. 15, 104075 (2020). 
36. Foley, J. Outdoor Recreation, Greenspace, and COVID-19 in Maine. Muskie School Capstones and Dissertations(2020).

37. CDC. COVID-19 and Your Health. Centers for Disease Control and Prevention https://www.cdc.gov/coronavirus/2019-ncov/daily-life-coping/participate-in-activities.html (2020).

38. Abouk, R. \& Heydari, B. The Immediate Effect of COVID-19 Policies on Social-Distancing Behavior in the United States. Public Health Rep. 0033354920976575 , https://doi.org/10.1177/0033354920976575 (2021).

39. Geng, D., Christina), Innes, J., Wu, W., Wang, G. \& (, \& Impacts of COVID-19 pandemic on urban park visitation: a global analysis. J. For. Res. 32, 553-567 (2021).

40. Hamidi, S. \& Zandiatashbar, A. Compact development and adherence to stay-at-home order during the COVID-19 pandemic: A longitudinal investigation in the United States. Landscape and Urban Planning. 205, 103952 (2021).

41. Connally, N. P. et al. Peridomestic Lyme disease prevention: results of a population-based casecontrol study. Am J Prev Med. 37, 201-206 (2009).

42. Cavany, S. M., España, G., Vazquez-Prokopec, G. M., Scott, T. W. \& Perkins, T. A. The impacts of COVID-19 mitigation on dengue virus transmission: a modelling study. medRxiv 2020.11.17.20210211(2020) doi:10.1101/2020.11.17.20210211.

43. Lim, J. T. et al. Increased Dengue Transmissions in Singapore Attributable to SARS-CoV-2 Social Distancing Measures. The Journal of Infectious Diseases. 223, 399-402 (2021).

44. Google COVID-19 Community Mobility Report. COVID-19 Community Mobility Report https://www.google.com/covid19/mobility?hl=es.

45. Rice, W. L. \& Pan, B. Understanding drivers of change in park visitation during the COVID-19 pandemic: A spatial application of Big data. (2020) doi:10.31235/osf.io/97qa4.

46. Rice, W. L. et al. Longitudinal changes in the outdoor recreation community's reaction to the COVID19 pandemic: Final report on a three-phase national survey of outdoor enthusiasts. (2020) doi:10.31235/osf.io/gnjcy.

47. Rice, W. L. et al. Changes in recreational behaviors of outdoor enthusiasts during the COVID-19 pandemic: analysis across urban and rural communities. Journal of Urban Ecology6, (2020).

48. Landry, C. E., Bergstrom, J., Salazar, J. \& Turner, D. How Has the COVID-19 Pandemic Affected Outdoor Recreation in the U.S.? A Revealed Preference Approach. Applied Economic Perspectives and Policy. 43, 443-457 (2021).

49. Randler, C., Tryjanowski, P., Jokimäki, J., Kaisanlahti-Jokimäki, M. L. \& Staller, N. SARS-CoV2 (COVID19) Pandemic Lockdown Influences Nature-Based Recreational Activity: The Case of Birders. International Journal of Environmental Research and Public Health. 17, 7310 (2020).

50. Bell, S. L., Phoenix, C., Lovell, R. \& Wheeler, B. W. Green space, health and wellbeing: making space for individual agency. Health \& Place. 30, 287-292 (2014). 
51. Pouso, S. et al. Contact with blue-green spaces during the COVID-19 pandemic lockdown beneficial for mental health. Science of The Total Environment. 756, 143984 (2021).

\section{Figures}

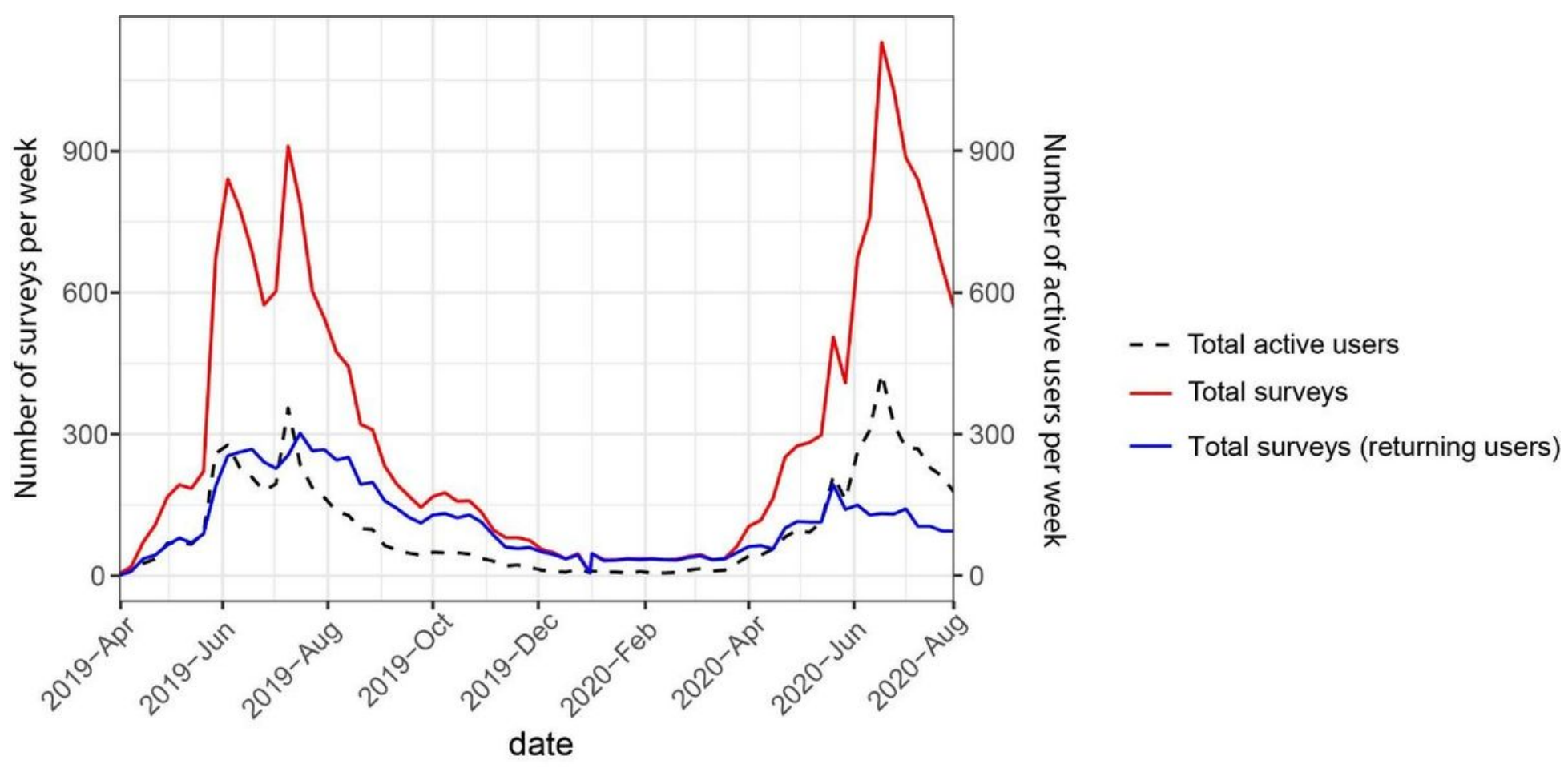

\section{Figure 1}

For a given week, the total number of surveys submitted and the total number of surveys submitted by returning users (those who participated in 2019 and 2020) (Left y-axis). For a given week, the number of total active users (those that submitted at least one survey) (Right y-axis). 


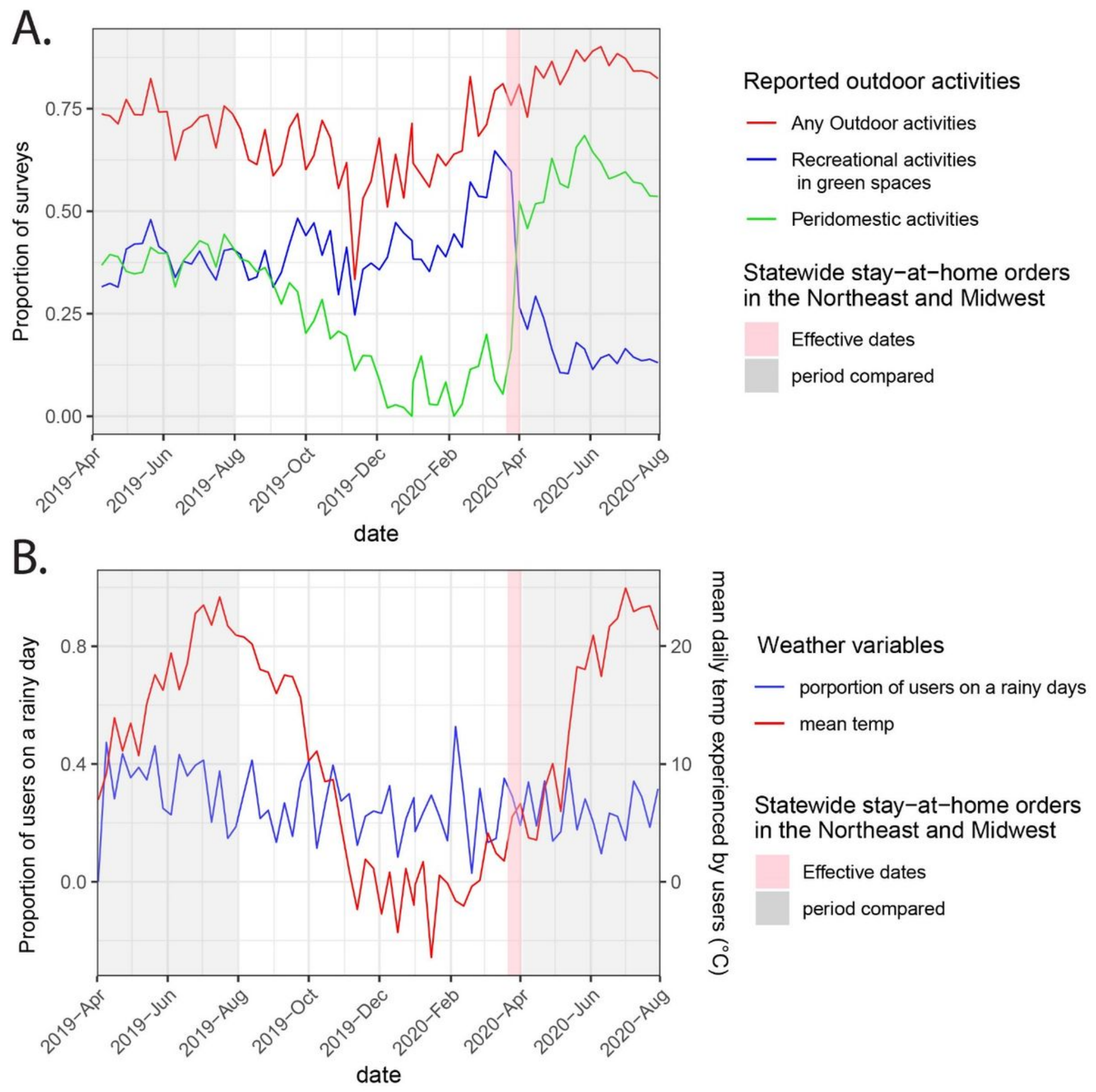

Figure 2

Unadjusted proportion of surveys reporting any type of outdoor activities, peridomestic activities or recreational activities in green spaces (parks and natural areas), per week (A). Weather variables experienced by users (weekly medians) (B). Weeks shaded in light grey indicate the time period that was compared between 2019 and 2020. Weeks shaded in light red indicate the effective dates for stay-athome orders in Northeast and Midwest counties. 


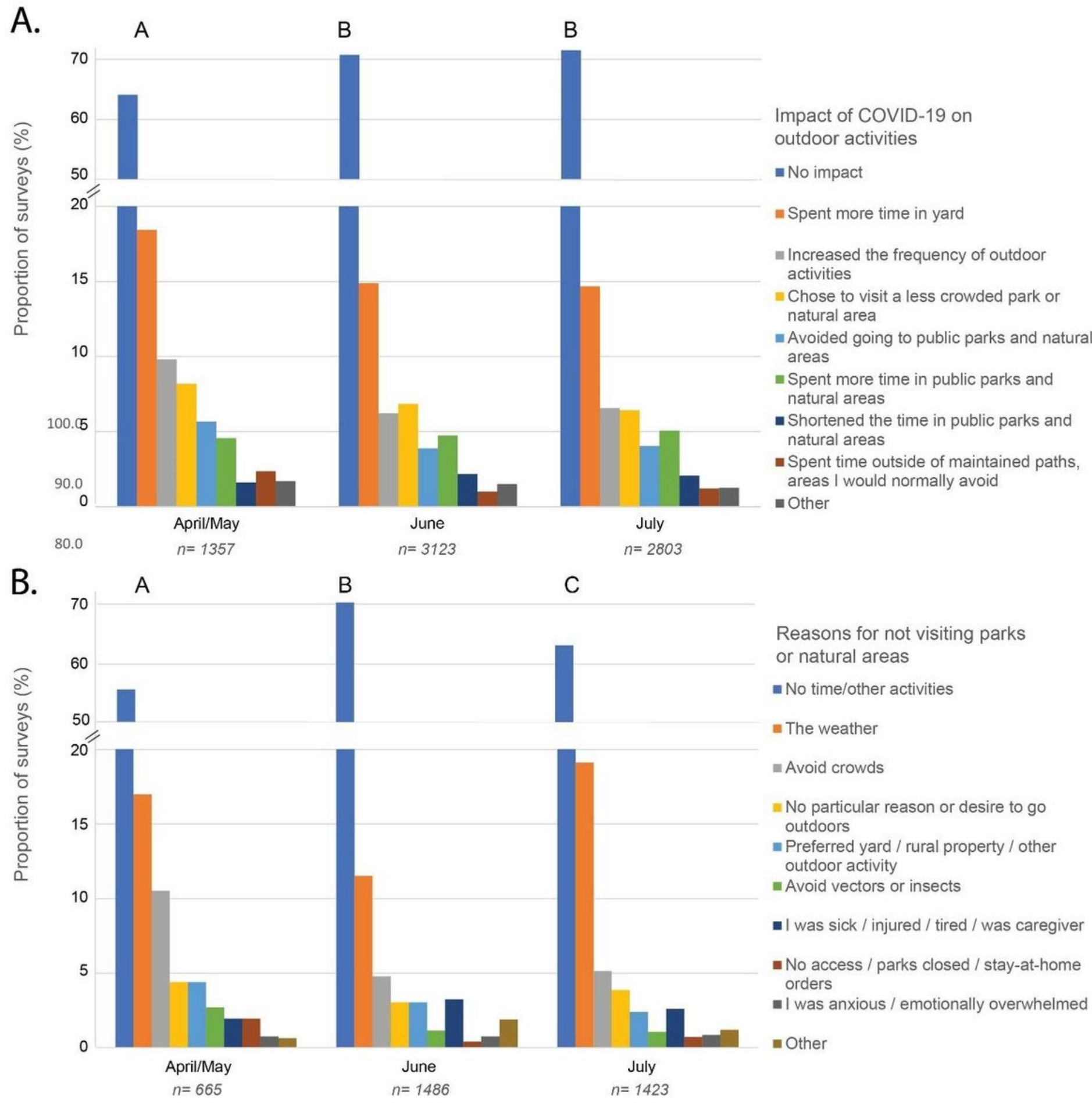

\section{Figure 3}

Self-reported impacts of COVID-19 on outdoor activities (A) and the reason for not having visited a park or recreational area that day (B). Different letters above the distributions (A-B-C) indicate significant differences $(X 2$ test, $P<0.001)$ between pair-wise comparisons between months after applying a Bonferroni correction for multiple comparisons. 


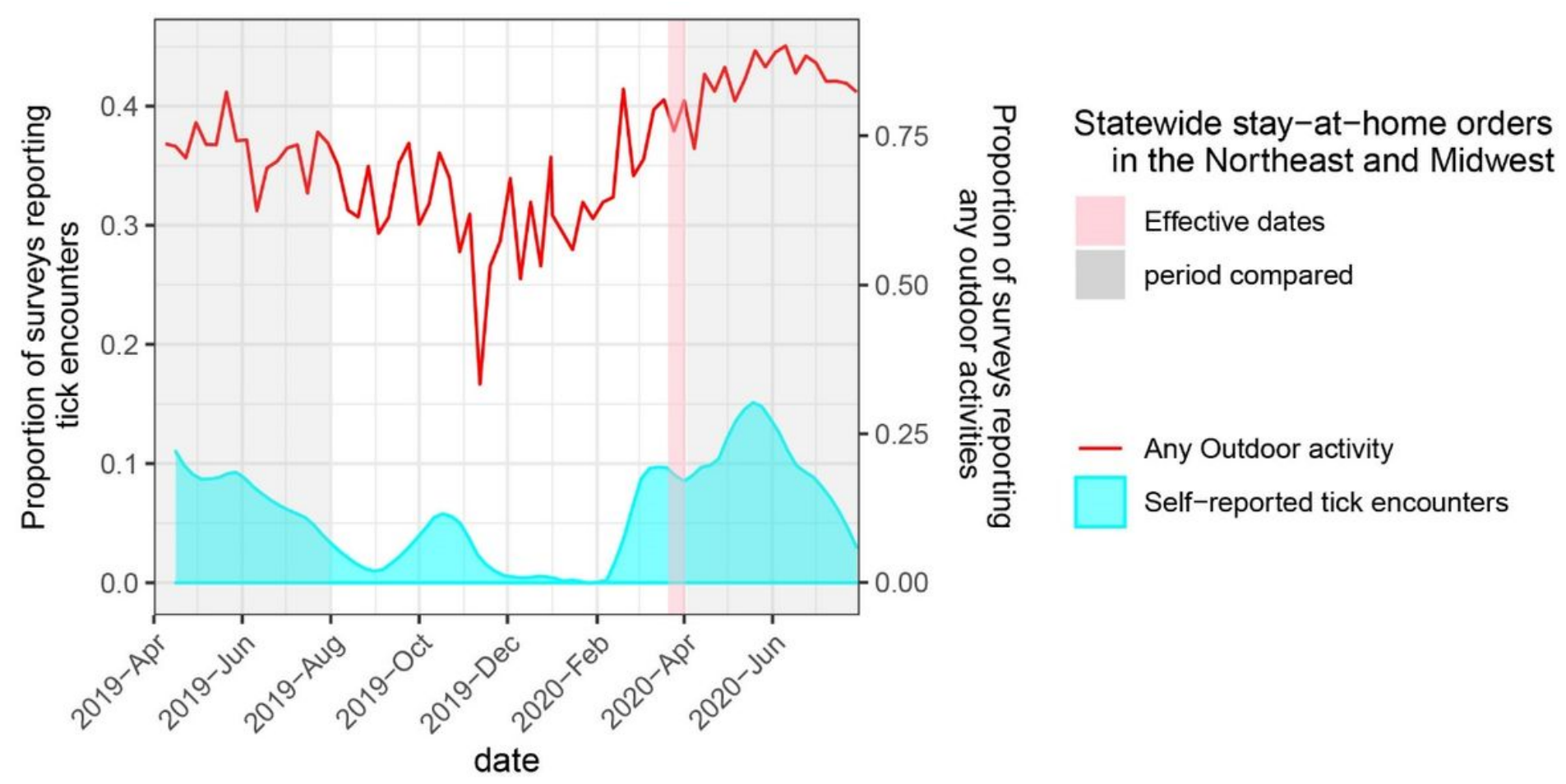

\section{Figure 4}

For a given week, we show the unadjusted proportion of surveys reporting at least one tick encounter (any tick species) (Left y-axis). For a given week, we show the unadjusted proportion of surveys reporting any outdoor activity (Right y-axis). Weeks shaded in light grey indicate the time period that was compared between 2019 and 2020. Weeks shaded in light red indicate the effective dates for stay-at-home orders in Northeast and Midwest counties.

\section{Supplementary Files}

This is a list of supplementary files associated with this preprint. Click to download.

- SupplementaryMaterialsFernandezetal.pdf 